\title{
P-Wave Arrival Times for the 1991 Racha, Georgia Earthquake Sequence at Stations of a Test, Sparse Network
}

S. C. Myers, C. A. Schultz, F. Ryall

February 2, 2000

U.S. Department of Energy

Lawrence

Livermore

National

Laboratory 


\section{DISCLAIMER}

This document was prepared as an account of work sponsored by an agency of the United States Government. Neither the United States Government nor the University of California nor any of their employees, makes any warranty, express or implied, or assumes any legal liability or responsibility for the accuracy, completeness, or usefulness of any information, apparatus, product, or process disclosed, or represents that its use would not infringe privately owned rights. Reference herein to any specific commercial product, process, or service by trade name, trademark, manufacturer, or otherwise, does not necessarily constitute or imply its endorsement, recommendation, or favoring by the United States Government or the University of California. The views and opinions of authors expressed herein do not necessarily state or reflect those of the United States Government or the University of California, and shall not be used for advertising or product endorsement purposes.

This work was performed under the auspices of the U.S. Department of Energy by the University of California, Lawrence Livermore National Laboratory under Contract No. W-7405-Eng-48.

This report has been reproduced directly from the best available copy.

Available to DOE and DOE contractors from the Office of Scientific and Technical Information

P.O. Box 62, Oak Ridge, TN 37831

Prices available from (423) 576-8401

http://apollo.osti.gov/bridge/

Available to the public from the National Technical Information Service

U.S. Department of Commerce 5285 Port Royal Rd., Springfield, VA 22161 http://www.ntis.gov/

OR

Lawrence Livermore National Laboratory Technical Information Department's Digital Library http://www.llnl.gov/tid/Library.html 


\title{
P-wave Arrival Times for the 1991 Racha, Georgia Earthquake Sequence at Stations of a Test, Sparse Network
}

\author{
Stephen C. Myers, Craig A. Schultz, and Floriana Ryall \\ Geophysics and Global Security \\ Lawrence Livermore National Laboratory \\ L-205, P.O. Box 808, Livermore, CA 94551
}

This work was Performed under the auspices of the U.S. Department of Energy by Lawrence Livermore National Laboratory under contract No. W-7405-Eng-48 for the office of Research and Development, NN-20, within the Office of NonProliferation and National Security, NNI. 


\section{Summary}

The following arrival information is a supplement to Myers and Schultz (2000). Myers and Schultz (2000) demonstrate the improvement in sparse-network location that can be achieved by using travel-time corrections determined with a Bayesian Kriging algorithm (Schultz et al., 1998). Precise, benchmark locations are provided by a local aftershock study of the 1991 Racha, Georgia earthquake sequence in the Caucasus Mountains (Fuenzalida et al., 1997). A test network is used to relocate the aftershocks with and without travel-time corrections. The test network is meant to represent a typical International Monitoring System configuration, with 6 stations at regional to near teleseismic distances (less then $30^{\circ}$ from the epicenter). The following arrival-time data help to facilitate the reproduction of Myers and Schultz (2000).

The arrival picks were obtained from the International Seismic Center (ISC) (openly available) and a Lawrence Livermore National Laboratory (LLNL) analyst (Flori Ryall). Table 1 lists the arrivals in epic time (time since January 1, 1970). The author of the arrival pick is listed as either "flori" or "_", where "_" indicates ISC. Table 2 lists the hypocenter information determined in the local aftershock study of Fuenzalida et al. (1997), and Table 3 lists the station information for the Racha test network. Fields in all tables are described in the CSS3.0 database schema.

\section{References}

Fuenzalida, H., L. Rivera, H. Haessler, D. LeGrand, H. Philip, L. Dorbath, D. McCormack, S. Arefiev, C. Langer, and A. Cisternas (1997) Seismic source study of the Racha-Dzhava (Georgia) earthquake from aftershocks and broad-band teleseismic body-wave records: an example of active nappe tectonics, Geophys. J. Int., v. 130, 29-46

Myers, S.C. and C. A. Schultz, Improving sparse-network seismic location with Bayesian Kriging and teleseismically constrained calibration events, Bull. Seism. Soc. Am., In press.

Schultz, C.A., S.C. Myers, J. Hipp, C. Young (1998). Nonstationary Bayesian kriging: a predictive technique to generate corrections for detection, location, and discrimination, Bull. Seism. Soc. Am., 88, 1275-1288. 
Table 1

Racha Arrival-Time Picks

\begin{tabular}{|c|c|c|}
\hline ORID & \multicolumn{2}{|c|}{ IPHASE TIME AUTH } \\
\hline $91119016 \mathrm{KVT}$ & $\mathrm{P}$ & $672916454.200-$ \\
\hline 91119016 SVE & $\mathrm{P}$ & $672916615.000-$ \\
\hline 91119016 GAR & $\mathrm{P}$ & $672916646.000-$ \\
\hline $91119016 \mathrm{KHO}$ & $\mathrm{P}$ & $672916662.700-$ \\
\hline 91119016 ARU & $\mathrm{P}$ & 672916600.861 flori \\
\hline $91119041 \mathrm{KVT}$ & $\mathrm{P}$ & $672939011.300-$ \\
\hline $91119041 \mathrm{SVE}$ & $\mathrm{P}$ & $672939175.000-$ \\
\hline $91119041 \mathrm{ARU}$ & $\mathrm{P}$ & 672939161.463 flori \\
\hline $91119041 \mathrm{KHO}$ & $\mathrm{P}$ & $672939223.200-$ \\
\hline $91119049 \mathrm{KAS}$ & $\mathrm{P}$ & $672945136.000-$ \\
\hline 91119049 ARU & $\mathrm{P}$ & 672945262.344 flori \\
\hline $91119049 \mathrm{SVE}$ & $\mathrm{P}$ & $672945276.000-$ \\
\hline $91119049 \mathrm{KHO}$ & $\mathrm{P}$ & $672945324.000-$ \\
\hline $91119056 \mathrm{KVT}$ & $\mathrm{P}$ & $672949929.700-$ \\
\hline 91119056 ARU & $\mathrm{P}$ & 672950073.380 flori \\
\hline $91119056 \mathrm{SVE}$ & $P$ & $672950088.000-$ \\
\hline 91119056 GAR & $\mathrm{P}$ & $672950120.000-$ \\
\hline $91119056 \mathrm{KHO}$ & $\mathrm{P}$ & $672950133.000-$ \\
\hline $91119063 \mathrm{KVT}$ & $\mathrm{P}$ & $672956016.300-$ \\
\hline $91119063 \mathrm{KAS}$ & $\mathrm{P}$ & $672956041.000-$ \\
\hline 91119063 ARU & $\mathrm{P}$ & 672956160.241 flori \\
\hline $91119063 \mathrm{KHO}$ & $\mathrm{P}$ & $672956220.000-$ \\
\hline $91120026 \mathrm{KVT}$ & $\mathrm{P}$ & $673015572.100-$ \\
\hline $91120026 \mathrm{KAS}$ & $\mathrm{P}$ & $673015595.400-$ \\
\hline 91120026 ARU & $\mathrm{P}$ & 673015720.557 flori \\
\hline $91120030 \mathrm{KVT}$ & $\mathrm{P}$ & $673024831.700-$ \\
\hline $91120030 \mathrm{KAS}$ & $\mathrm{P}$ & $673024861.500-$ \\
\hline $91120030 \mathrm{ARU}$ & $\mathrm{P}$ & 673024980.506 flori \\
\hline $91120030 \mathrm{GAR}$ & $\mathrm{P}$ & $673025025.700-$ \\
\hline $91120036 \mathrm{KVT}$ & $\mathrm{P}$ & $673027743.700-$ \\
\hline 91120036 ARU & $\mathrm{P}$ & 673027894.622 flori \\
\hline 91120036 SVE & $P$ & $673027910.000-$ \\
\hline 91120036 GAR & $\mathrm{P}$ & $673027943.000-$ \\
\hline $91120036 \mathrm{KHO}$ & $\mathrm{P}$ & $673027956.000-$ \\
\hline 91121009 SVE & $\mathrm{P}$ & $673069341.800-$ \\
\hline 91121009 GAR & $\mathrm{P}$ & $673069373.000-$ \\
\hline $91121009 \mathrm{KHO}$ & $\mathrm{P}$ & $673069387.000-$ \\
\hline 91121009 ARU & $\mathrm{P}$ & 673069326.785 flori \\
\hline $91121040 \mathrm{KVT}$ & $\mathrm{P}$ & $673106774.600-$ \\
\hline $91121040 \mathrm{SVE}$ & $\mathrm{P}$ & $673106936.000-$ \\
\hline
\end{tabular}




\begin{tabular}{|c|c|c|}
\hline 91121040 ARU & $\mathrm{P}$ & 673106922.547 flori \\
\hline $91121040 \mathrm{KHO}$ & $\mathrm{P}$ & $673106984.000-$ \\
\hline $91121041 \mathrm{KVT}$ & $\mathrm{P}$ & $673108759.600-$ \\
\hline $91121041 \mathrm{KHO}$ & $\mathrm{P}$ & $673108973.000-$ \\
\hline $91121041 \mathrm{ARU}$ & $\mathrm{P}$ & 673108909.156 flori \\
\hline $91121054 \mathrm{SVE}$ & $\mathrm{P}$ & $673140198.000-$ \\
\hline 91121054 GAR & $\mathrm{P}$ & $673140230.100-$ \\
\hline 91121054 ARU & $\mathrm{P}$ & 673140183.847 flori \\
\hline $91121054 \mathrm{KHO}$ & $\mathrm{P}$ & $673140244.000-$ \\
\hline $91123004 \mathrm{KVT}$ & $\mathrm{P}$ & $673251012.000-$ \\
\hline 91123004 ARU & $\mathrm{P}$ & 673251153.892 flori \\
\hline $91123004 \mathrm{KAS}$ & $\mathrm{P}$ & $673251024.800-$ \\
\hline 91123004 GAR & $\mathrm{P}$ & $673251200.000-$ \\
\hline $91123004 \mathrm{KHO}$ & $\mathrm{P}$ & $673251214.000-$ \\
\hline $91123025 \mathrm{KVT}$ & $\mathrm{P}$ & $673302062.500-$ \\
\hline $91123025 \mathrm{KAS}$ & $\mathrm{P}$ & $673302084.800-$ \\
\hline 91123025 SVE & $P$ & $673302229.000-$ \\
\hline 91123025 GAR & $\mathrm{P}$ & $673302262.000-$ \\
\hline $91123025 \mathrm{KHO}$ & $\mathrm{P}$ & $673302279.000-$ \\
\hline 91123025 ARU & $\mathrm{P}$ & 673302212.902 flori \\
\hline $91134023 \mathrm{KAS}$ & $\mathrm{P}$ & $674213895.000-$ \\
\hline 91134023 SVE & $\mathrm{P}$ & $674214033.000-$ \\
\hline 91134023 ARU & $\mathrm{P}$ & 674214019.324 flori \\
\hline 91134023 GAR & $\mathrm{P}$ & $674214067.000-$ \\
\hline $91135030 \mathrm{KVT}$ & $\mathrm{P}$ & $674317814.500-$ \\
\hline $91135030 \mathrm{ARU}$ & $\mathrm{P}$ & 674317966.062 flori \\
\hline $91135030 \mathrm{KAS}$ & $\mathrm{P}$ & $674317837.100-$ \\
\hline 91135030 SVE & $\mathrm{P}$ & $674317980.000-$ \\
\hline $91135030 \mathrm{KHO}$ & $\mathrm{P}$ & $674318029.000-$ \\
\hline $91135030 \mathrm{GAR}$ & $\mathrm{P}$ & 674318012.889 flori \\
\hline $91143012 \mathrm{KVT}$ & $\mathrm{P}$ & $674988177.000-$ \\
\hline 91143012 ARU & $\mathrm{P}$ & 674988331.673 flori \\
\hline 91143012 GAR & $\mathrm{P}$ & 674988376.917 flori \\
\hline $91143012 \mathrm{KAS}$ & $\mathrm{P}$ & $674988201.000-$ \\
\hline 91143012 SVE & $\mathrm{P}$ & $674988343.000-$ \\
\hline $91143012 \mathrm{KHO}$ & $\mathrm{P}$ & $674988391.000-$ \\
\hline $91144013 \mathrm{KVT}$ & $\mathrm{P}$ & $675072059.400-$ \\
\hline $91144013 \mathrm{KAS}$ & $\mathrm{P}$ & $675072083.000-$ \\
\hline 91144013 GAR & $\mathrm{P}$ & 675072253.973 flori \\
\hline 91144013 ARU & $\mathrm{P}$ & 675072207.389 flori \\
\hline $91144013 \mathrm{SVE}$ & $\mathrm{P}$ & $675072223.000-$ \\
\hline
\end{tabular}


Table 2

Racha Ground-Truth Locations

$\begin{array}{lcccccc}\text { ORID } & \text { LAT } & \text { LON } & \text { DEPTH } & \text { TIME } & \text { JDATE } \\ 91119016 & 42.4220 & 43.6950 & 7.000 & 672916366.400 & 1991119 \\ 91119041 & 42.4530 & 43.5150 & 7.000 & 672938925.800 & 1991119 \\ 91119049 & 42.4510 & 43.5660 & 7.000 & 672945027.500 & 1991119 \\ 91119056 & 42.4560 & 43.9050 & 13.200 & 672949841.100 & 1991119 \\ 91119063 & 42.4350 & 43.8880 & 7.000 & 672955926.200 & 1991119 \\ 91120026 & 42.5240 & 43.5110 & 8.700 & 673015486.200 & 1991120 \\ 91120030 & 42.4370 & 43.8530 & 7.000 & 673024746.800 & 1991120 \\ 91120036 & 42.5290 & 43.3130 & 7.000 & 673027659.700 & 1991120 \\ 91121009 & 42.5600 & 43.4470 & 2.600 & 673069091.600 & 1991121 \\ 91121040 & 42.5510 & 43.4740 & 11.100 & 673106687.900 & 1991121 \\ 91121041 & 42.5710 & 43.3990 & 7.000 & 673108674.700 & 1991121 \\ 91121054 & 42.4350 & 43.9240 & 11.400 & 673139950.300 & 1991121 \\ 91123004 & 42.5130 & 43.3690 & 7.000 & 673250917.300 & 1991123 \\ 91123025 & 42.5840 & 43.2460 & 7.700 & 673301979.600 & 1991123 \\ 91134023 & 42.5190 & 43.5330 & 4.500 & 674213784.900 & 1991134 \\ 91135030 & 42.4930 & 43.3850 & 4.300 & 674317730.200 & 1991135 \\ 91143012 & 42.4810 & 43.2730 & 2.300 & 674988092.600 & 1991143 \\ 91144013 & 42.4100 & 43.6670 & 2.300 & 675071971.600 & 1991144\end{array}$

Table 3

Racha Site Information

$\begin{array}{lccl}\text { STA } & \text { LAT } & \text { LON } & \text { ELEV(KM) } \\ \text { ARU } & 56.4000 & 58.6000 & .0000 \\ \text { GAR } & 39.0000 & 70.3167 & 1.0000 \\ \text { KAS } & 41.3717 & 33.7667 & .0000 \\ \text { KHO } & 37.4833 & 71.5333 & 1.0000 \\ \text { KVT } & 41.0806 & 36.0464 & .0000 \\ \text { SVE } & 56.8100 & 60.6367 & .0000\end{array}$

